TORUS: Theory of Reactions for Unstable iSotopes Annual Continuation and Progress Report Year-2: March 1, 2011 - February 29, 2012

G. Arbanas, C. Elster, J. Escher, A. Mukhamedzanov, F. Nunes, I. J. Thompson

February 27, 2012 
This document was prepared as an account of work sponsored by an agency of the United States government. Neither the United States government nor Lawrence Livermore National Security, LLC, nor any of their employees makes any warranty, expressed or implied, or assumes any legal liability or responsibility for the accuracy, completeness, or usefulness of any information, apparatus, product, or process disclosed, or represents that its use would not infringe privately owned rights. Reference herein to any specific commercial product, process, or service by trade name, trademark, manufacturer, or otherwise does not necessarily constitute or imply its endorsement, recommendation, or favoring by the United States government or Lawrence Livermore National Security, LLC. The views and opinions of authors expressed herein do not necessarily state or reflect those of the United States government or Lawrence Livermore National Security, LLC, and shall not be used for advertising or product endorsement purposes.

This work performed under the auspices of the U.S. Department of Energy by Lawrence Livermore National Laboratory under Contract DE-AC52-07NA27344. 
Office of Science, U.S. Department of Energy

Office of Nuclear Physics

Nuclear Theory Division

\title{
TORUS:
}

\section{Theory of Reactions for Unstable iSotopes}

\author{
A Topical Collaboration for Nuclear Theory \\ Project Period: June 1, 2010 - May 31, 2015
}

Annual Continuation and Progress Report

Year-2: March 1, 2011 - February 29, 2012

Prepared by the TORUS Collaboration:

Goran Arbanas

Charlotte Elster

Jutta Escher

Akram Mukhamedzhanov

Filomena Nunes

Ian Thompson (Coordinating P.I.)

February 24, 2012 


\section{Contents}

$\begin{array}{llr}1 & \text { Introduction } & 3\end{array}$

2 Topics $\quad 4$

2.1 Distinguishing Peripheral and Interior Contributions . . . . . . . . . . . . 4

2.2 Breakup and Transfer Models . . . . . . . . . . . . . . . . . . . 6

2.3 Generalized Faddeev Methods . . . . . . . . . . . . . . . . . . . 8

2.4 Transfers to Resonances . . . . . . . . . . . . . . . . . . . 9

2.5 Capture Reactions . . . . . . . . . . . . . . . . . . . 11

2.5.1 Semi-direct capture mechanisms . . . . . . . . . . . . 11

2.5.2 Identifying a need for better reactions model in nuclear astrophysics(?) . . 12

2.5.3 Two-hole pair structure of the ${ }^{130} \mathrm{Sn}$ ground state . . . . . . . . . . . 12

2.6 Isobaric Analogue Resonances . . . . . . . . . . . . . . . . . . . . 13

2.7 Pair Transfers . . . . . . . . . . . . . . . . . . . . . . 13

2.8 Implementing Advanced Optical Potentials . . . . . . . . . . . . . . . 13

2.8.1 Dispersive Optical Potentials . . . . . . . . . . . . . . . . . . 13

2.8.2 Microscopic Folding Optical Potentials . . . . . . . . . . . . . . . . . 14

2.8.3 Investigating Separable Optical Potentials for Faddeev Models . . . . . . . 15

3 Project Management $\quad 16$

4 Postdoctoral Staff and Visits $\quad 17$

5 Plans for Year $3 \quad 18$

6 Deliverables $\quad 19$

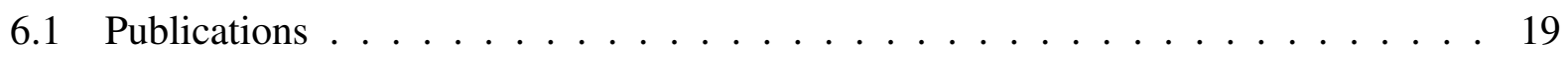

6.2 Preprints and Reports . . . . . . . . . . . . . . . . . 22

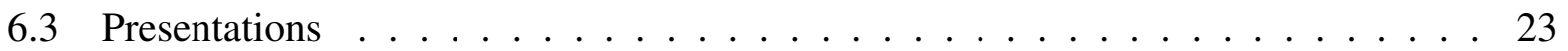

6.4 In Preparation . . . . . . . . . . . . . . . . . . . . . . 24

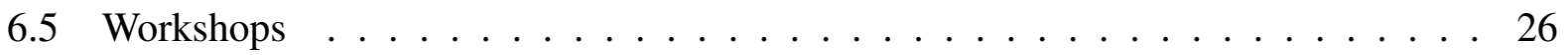

6.5 .1 Proposed TORUS/INT workshop . . . . . . . . . . . 26

$\begin{array}{ll}\text { Bibliography } & 27\end{array}$ 


\section{Introduction}

\section{Background}

The TORUS collaboration derives its name from the research it focuses on, namely the Theory of Reactions for Unstable iSotopes. It is a Topical Collaboration in Nuclear Theory, and funded by the Nuclear Theory Division of the Office of Nuclear Physics in the Office of Science of the Department of Energy. The funding supports one postdoctoral researcher for the years 1 through 3. The collaboration brings together as Principal Investigators a large fraction of the nuclear reaction theorists currently active within the USA.

\section{Mission}

The mission of the TORUS Topical Collaboration is to develop new methods that will advance nuclear reaction theory for unstable isotopes by using three-body techniques to improve directreaction calculations, and, by using a new partial-fusion theory, to integrate descriptions of direct and compound-nucleus reactions. This multi-institution collaborative effort is directly relevant to three areas of interest: the properties of nuclei far from stability; microscopic studies of nuclear input parameters for astrophysics, and microscopic nuclear reaction theory.

\section{Highlights from Year 2}

1. Detailed comparison of CDCC and Faddeev three-body calculations submitted by postdoc N. Upadhyay [29] - Section 2.2.

2. Discovery of new method for transfers to resonances [17], which suggests a surface approximation useful for experimental analysis of resonances - Section 2.4.

3. Tests of the for the surface approximation - Section 2.1.

4. Testing and establishment of 'finite-range adiabatic distorted wave method' for a deuteron stripping [21] - Section 2.2.

5. Trojan Horse method applied to astrophysical reaction ${ }^{19} \mathrm{~F}(\mathrm{p}, \alpha){ }^{16} \mathrm{O}[8]-$ Section 2.4 .

6. 11 papers published and another 4 submitted - Section 6.2.

7. Research done by the TORUS collaboration has been featured in invited talks at various national and international venues, including several ECT* workshops in Trento (4 talks), INT workshops in Seattle (3 talks), RIKEN meetings in Japan (2 talks), and a plenary talk at CGS14 in Guelph, Canada. 


\section{Topics}

New opportunities arising from research completed in Year 2, as well as DOE-imposed funding reductions have motivated us to change our research priorities for Years 3 and 4. Specifically, the newly-developed surface-integral formulation developed by A.M. Mukhamdezhanov (see Sections 2.1 and 2.4 below and Ref. [17]) has opened up the possibility to analyze stripping to resonance states in a new manner (see Section 2.4). We have already begun to test the underlying approximations (see Section 2.1) and plan to pursue this promising approach more fully in Year 3 (see Section 2.4). We are also pursuing a stepped-up effort to support the development of threebody (AGS) methods. Specifically, we plan to carry out additional work on separable potentials (Section 2.8.3) and work on momentum-space Faddeev methods for charged systems (Section 2.3).

To support these objectives in the context of an overall funding reduction, we find it necessary to move funds from LLNL and ORNL support to the Ohio and Texas A\&M Universities. The laboratories' budgets will be reduced by a total of $\$ 33 \mathrm{k}$. This is a one-time re-direction of support and we plan to resume the original distribution of funding between laboratories and universities in Year 4. As a consequence of the funding changes, we have to delay the work on partial fusion of deuterons incident on heavy targets. As this is an important aspect of the overall TORUS project, it will be pursued in Year 4 by Livermore Laboratory researchers Thompson and Escher. Similarly, we will delay the research on pygmy resonances, led by Arbanas, and postpone the pursuit of a workshop on $(\mathrm{d}, \mathrm{p})$ reactions.

\subsection{Distinguishing Peripheral and Interior Contributions}

\section{Personnel involved: A.M. Mukhamdezhanov, F.M. Nunes, J.E. Escher, and I.J. Thompson}

A crucial deliverable of any reaction theory is to determine what information can eventually be extracted from the analysis of the deuteron stripping data. It is well recognized by now that these reactions are mainly peripheral at energies $<15 \mathrm{MeV} /$ nucleon; the normalization of the external region is controlled by the asymptotic normalization constant (ANC). In 2011, A.M.M and F.M.N. contributed to the analysis of the astrophysically important reaction ${ }^{14} \mathrm{C}(\mathrm{d}, \mathrm{p}){ }^{15} \mathrm{C}$, which was measured, for deuteron energy $E_{d}=17 \mathrm{MeV}$, at the Nuclear Physics Institute, Prague-Rez, Czech Academy of Sciences, in collaboration with Texas A\&M University and MSU/NSCL. As this reaction is a prime candidate for applying the generalized AGS equations developed by the TORUS collaboration, it deserves particular attention. The target is $\beta$-unstable with two neutrons outside the ${ }^{12} \mathrm{C}$ core and the loosely bound ${ }^{14} \mathrm{C}+\mathrm{n}$ state is almost of single-particle nature. The direct radiative capture ${ }^{14} \mathrm{C}(\mathrm{n}, \gamma){ }^{15} \mathrm{C}$ plays an important role in nuclear astrophysics. The analysis of the reaction was carried out within the adiabatic DWA, which is a simplified version of the FRADWA, and a standard DWBA analysis was performed for comparison. A major conclusion of our study is that the ${ }^{14} \mathrm{C}(\mathrm{d}, \mathrm{p}){ }^{15} \mathrm{C}$ reaction can be used as a tool to determine the ANCs, which in turn provides important information for calculating the $(n, \gamma)$ cross section, but to obtain reliability and better accuracy it is necessary to go beyond DWBA. The findings are summarized in the paper [16].

Employing the new formalism developed by A. M. Mukhamedzhanov (see Section 2.4 and [17]), A.M.M., I.J.T., and J.E.E. began to examine the relative interior and exterior contributions 

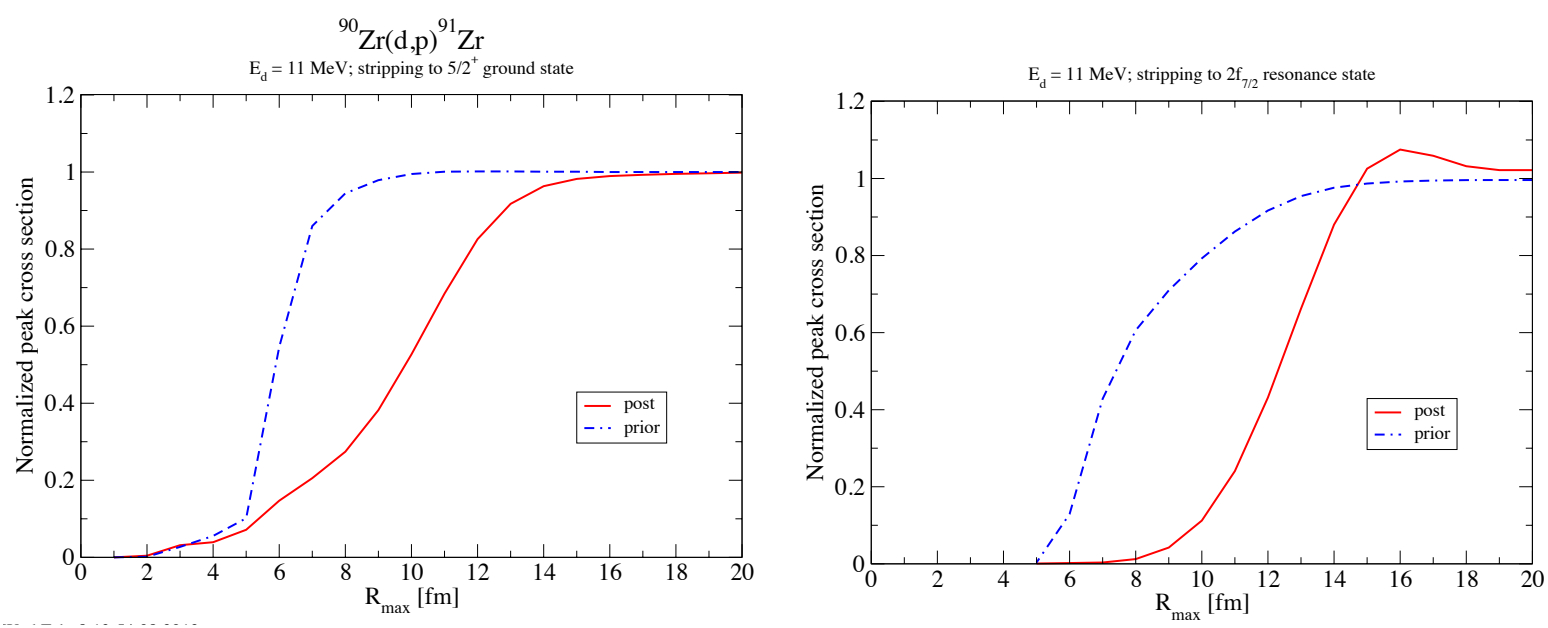

Figure 1: Examination of the role of exterior contributions for stripping to the ground state of ${ }^{91} \mathrm{Zr}$ (top panel) and a $2 f_{7 / 2}$ narrow resonance at $0.993 \mathrm{MeV}$ (bottom panel). Peak cross sections, calculated in the post and prior formalisms, are shown as a function of the cutoff radius $R_{\max }$, beyond which contributions from the reaction amplitude are set to zero. The cross sections are normalized relative to the peak cross sections obtained in the full calculation.

of the reaction amplitude to calculated transfer cross sections. We considered stripping to both bound and resonance states. The reaction code FRESCO was modified in order to allow us to investigate separately the contributions from the internal and external parts of the reaction amplitude, where internal and external is defined in terms of the distance $r_{n A}$ between the transferred neutron and the target. Calculations were carried out for a range of different targets, ${ }^{12} \mathrm{C},{ }^{16} \mathrm{O},{ }^{40} \mathrm{Ca},{ }^{90} \mathrm{Zr}$, and ${ }^{208} \mathrm{~Pb}$, for incident deuteron energies between 11 and $82 \mathrm{MeV}$. For now the analysis has been performed in the DWBA formalism, and will be extended to the coupled-channels approach. An example is shown in Figure 1. Our main finding is that, although the post and prior DWBA amplitudes are equal, their behavior is different in the subspace over the variable $r_{n A}$. While the post form is almost entirely peripheral at deuteron energies $E_{d} \leq 30 \mathrm{MeV}$, only the internal part contributes to the prior form. The fact that both forms nevertheless do coincide can be shown formally by transforming the volume integral to surface form in the subspace over the variable $\mathbf{r}_{n A}$. These results demonstrate clearly that stripping reactions at $E_{d} \leq 30 \mathrm{MeV}$ can be equated to capture only when they have a peripheral character. This, in turn, confirms that the asymptotic normalization coefficient (ANC) is the most relevant and appropriate quantity that can be determined from such experiments.

What is even more important is that this result opens up a possibility to analyze stripping to resonance states, what is the second part of our research. The modified FRESCO code made it possible to investigate the peripheral character of the post and prior forms of the DWBA for stripping to resonance states. J.E.E. and I.J.T carried out calculations for ${ }^{90} \mathrm{Zr}(d, p)^{91} \mathrm{Zr}$ at $E_{d}=11 \mathrm{MeV}$, populating narrow $2 f_{7 / 2}$ and broad $2 f_{5 / 2}$ resonances. The initial finding were similar to the result obtained for stripping to bound states: the prior form is dominated by the internal contribution while the post form is entirely peripheral. In the context of this work, we also studied the convergence of stripping calculations for narrow and broad resonances. Various descriptions, including bins and a regularization factor $\exp \left(-\alpha r_{n A}\right)$, where $\alpha \rightarrow 0$, are compared; we expect to be able 
to formulate more general recommendations for stripping calculations that involve resonances.

These findings pave the way for the development of an approach, and a code, that makes it possible to analyze deuteron stripping experiments that involve resonance states and to extract the observable neutron resonance widths. We expect to report our findings in a letter and in a regular, longer article.

Year 3 plan: In an extension of this work, we will determine the size of exterior prior and interior post contributions for transfers to a wide range of resonances, to predict the accuracy of the Surface Approximation method of Section 2.4. We want to confirm in particular the smallness of the exterior prior contributions within the Continuum Discretized Coupled-Channels (CDCC) approach.

This work will be carried out by J.E. Escher, I.J. Thompson, and A.M. Mukhamedzhanov.

\subsection{Breakup and Transfer Models}

Personnel involved: N. Upadhyay (postdoc), F.M. Nunes, and Ch. Elster

One of the most well established theory for direct nuclear reactions is the Continuum Discretized Coupled Channels (CDCC) method. It includes breakup to all orders by discretizing the projectile continuum into bins. Alternatively, the 3-body problem can be solved exactly within the Faddeev momentum space integral formalism (here denoted FAGS) which explicitly includes breakup and transfer channels to all orders.

With the aim of quantifying the accuracy of CDCC in computing elastic, breakup and transfer cross sections and establish a range of validity, we have completed a systematic comparison of CDCC and Faddeev. Our test cases consist of: i) d $+{ }^{10} \mathrm{Be}$ at $E_{\mathrm{d}}=21.4,40.9$ and $71 \mathrm{MeV}$; ii) d $+{ }^{12} \mathrm{C}$ at $E_{\mathrm{d}}=12$ and $56 \mathrm{MeV}$; and iii) $\mathrm{d}+{ }^{48} \mathrm{Ca}$ at $E_{\mathrm{d}}=56 \mathrm{MeV}$. These reactions were chosen to match available experimental data however the goal of the project was to understand the limitations of CDCC and therefore no fine tuning of interactions was performed. This work has been submitted to publication (arXiv:1112.5338). As we summarize below, our results pose important constraints on the validity of CDCC when applied to deuteron induced reactions and call for a better description of the reaction dynamics.

We compute elastic scattering, transfer cross sections to the ground state of the final system, as well as breakup observables. In CDCC, elastic scattering and breakup cross sections are obtained directly from the S-matrix, while transfer is calculated replacing the exact three-body wavefunction by the CDCC wavefunction in the exact post-form T-matrix.

Our CDCC/FAGS comparisons show no immediate correlation between elastic, transfer or breakup. In other words, finding agreement for the elastic for a given target and beam energy does not imply agreement in breakup or transfer. Indeed, these processes are sensitive to different parts of configuration space and therefore, only by looking at elastic, transfer and breakup simultaneously, can the CDCC method be thoroughly tested.

Overall, and regardless of the beam energy, CDCC is able to provide a good approximation to FAGS for elastic scattering. The inclusion of a neutron-nucleus bound state in the FAGS1 calculations introduces small modifications mostly are backward angles. Only for $\mathrm{d}+{ }^{12} \mathrm{C}$ at $12 \mathrm{MeV}$ we found stronger discrepancies in the elastic angular distribution between CDCC and Faddeev-AGS. 


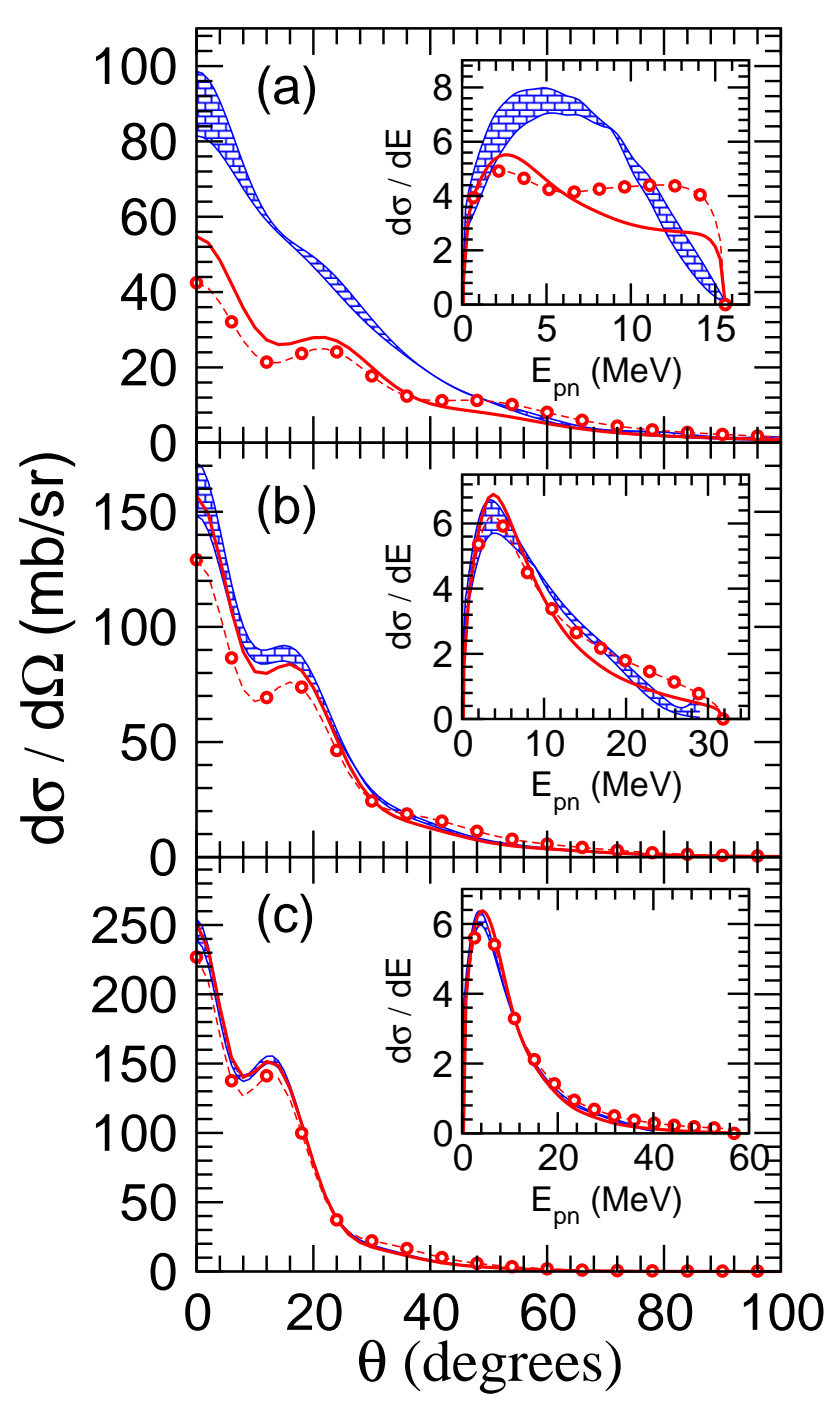

Figure 2: Breakup distributions for ${ }^{10} \mathrm{Be}\left(\mathrm{d}\right.$, pn) ${ }^{10} \mathrm{Be}$ reaction at: (a) $E_{\mathrm{d}}=21 \mathrm{MeV}$, (b) $E_{\mathrm{d}}=40.9 \mathrm{MeV}$ and (c) $E_{\mathrm{d}}=71 \mathrm{MeV}$. Results for CDCC (hashed bar), FAGS(solid) and FAGS1 (circles).

The comparison of CDCC and Faddeev-AGS for transfer cross sections is consistent with the results presented in [21]. We found CDCC to be a very good approximation of FAGS1 at reactions around $10 \mathrm{MeV} / \mathrm{u}$, but not so good for larger beam energies. What became clear from our study is that, for loosely bound s-wave dominated projectiles, CDCC does not improve the description of transfer when compared to the adiabatic model (ADWA). Because CDCC is computationally expensive, ADWA should be the preferred tool. Why, at larger beam energies, CDCC is not able to improve the description of breakup when compared to ADWA is not yet understood and would require a more detailed study.

Breakup observables predicted by CDCC are at its best for the higher beam energies explored in this work. To reduce the technical challenges of the problem, we ignore the Coulomb interaction in the breakup comparison. Also, we use exactly the same Hamiltonian (CDCC and FAGS) to remove any ambiguity.

In Fig. 2 we present the results for the angular distribution as a function of the c.m. angle of the $p n$ system following the breakup on ${ }^{10} \mathrm{Be}$ at the three energies of choice. At the lowest energy, 
we find that CDCC does not reproduce FAGS, even taking into account the error estimated by model space truncation. At the higher energies, this discrepancy is removed. The insets of Fig. 2 contain the corresponding energy distributions as functions of the proton-neutron relative energy $E_{p n}$. Again, a very large discrepancy is found at $21.4 \mathrm{MeV}$ while fair agreement between CDCC and FAGS is obtained at the higher energies.

Similar conclusions can be drawn from the comparison of breakup angular and energy distributions for reactions on ${ }^{12} \mathrm{C}$. Despite the large error bar in the CDCC predictions, there is a striking mismatch between CDCC and FAGS in both magnitude and shape of the breakup cross sections at $12 \mathrm{MeV}$. These discrepancies disappear at the higher energy. Agreement is obtained between CDCC and FAGS for the breakup of deuterons on ${ }^{48} \mathrm{Ca}$ at $56 \mathrm{MeV}$.

Although the calculations themselves represent a challenge, understanding the reason for the differences turns out to be even more challenging. We explored the effects of including the $\mathrm{nA}$ bound state in the transfer channel with FAGS1 (dotted circles). By comparing FAGS and FAGS1 we conclude that the effects of transfer are not negligible on breakup, particularly at low energies.

In addition we looked at the various components in the Faddeev approach. Strong contributions from the proton and neutron Faddeev components, not explicitly included in the CDCC expansion, are present when the proton-neutron relative energies are large. At low energy, the energy distribution is broad, the breakup to scattering states with large proton-neutron relative energy is important and therefore CDCC does not perform well. One possible solution to this shortcoming may be to use the CDCC wavefunction in a T-matrix that probes only short distances between the proton and neutron, instead of its asymptotic form. This work was submitted to Phys. Rev. C [29].

\subsection{Generalized Faddeev Methods}

\section{Personnel involved: N. Upadhyay (postdoc), Ch. Elster, A. Mukhamedzhanov and F.M. Nunes}

The comparative work discussed in Section 2.2 poses serious questions on the applicability of CDCC in various regimes. However, it is critical to realize that the number of reactions which can be calculated with the present implementation of Faddeev-AGS is very limited, particularly having in mind the reach of FRIB. For targets with charge $Z>20$, Coulomb effects require larger screening radius and more partial waves; and this renders the solution of the AGS equations in the partial-wave representation impossible, as done in [21]. In addition, the Padé summation technique used to solve the AGS equations converges slower at low energies $(10 \mathrm{MeV} / \mathrm{u})$, where couplings are strong, and it is not possible to obtain converged solutions even for mass $A=48$. It is exactly due to these limitations that TORUS will extend present techniques used in Faddeev-AGS and develop new methods to overcome those difficulties.

We use as starting point the Faddeev equations written in the commonly used Alt-GrassbergerSandhas (AGS) formulation. However, these equations are not sufficient to take into account the whole complexity of the deuteron stripping reactions, since in the Faddeev approach the target is treated as a structureless constituent. In Ref. [1] the AGS equations are generalized to matrix AGS equations, which take into account target excitations. The effective potentials are given by nucleon transfer diagrams, triangle exchange diagrams describing transfer, and triangle diagrams describing elastic and inelastic scattering. Because eventually we are going to apply the generalized equations for analysis of the deuteron stripping on targets with $A \geq 100$, two main obstacles should be 
resolved: stability of the screening procedure for a large charge of the target and the calculation of the triangle diagrams, which contain the off-shell Coulomb scattering amplitude. We already showed that the exchange triangle diagram can be approximately reduced to the renormalized pole diagram, which then can decrease the number of the effective potentials in the AGS equations.

The current year has been truly exploratory. We have established weekly meetings via internet between the three PIs and the post-doc to discuss progress and various aspects of the problems. In addition we have used this year to establish contact with various experts in the field: In October we held a small workshop with our visitors, Profs. Ron Johnson and George Rawitscher to discuss pros and cons of the various approaches. During her visit in Lisbon, Nunes discussed with Arnas Deltuva aspects of his implementation of the Faddeev method.

Out of this grew a detailed plan to proceed with our implementation of the AGS scheme for (d,p) reactions. The AGS equations will be solved using $N N$ and $N A$ potentials that reproduce corresponding elastic scattering phase shifts accurately in the energy regime under consideration, as well as the bound state poles. We will use separable representations of nucleon-nucleon (NN) and nucleon-nucleus (NA) interactions, since they reduce the dimension of the integrations in the Faddeev kernel and leave the AGS equations as large set of one dimensional coupled integral equations. Particular care and effort will have to be placed on the incorporation of the Coulomb interaction, especially triangle diagrams in the rearrangement, inelastic and elastic channels.

Year 3 plan: we will investigate whether the rearrangement triangle diagram containing the off-shell proton-target Coulomb scattering amplitude can be replaced by the renormalized pole diagram describing the proton transfer.

We will focus our effort on the treatment and practical implementation of the Coulomb potential in the AGS equations. It is standard to use a screened Coulomb potential. The main issue here is numerical stability when the screening radius is increased. We will exploit a screening procedure proposed by Oryu [23] and the Alt-Sandhas formulation of Coulomb effects in the three-body reactions with charged particles [2], which avoids a screening procedure solving the AGS equations for the Coulomb-modified short-range amplitudes.

We will start the actual coding of the AGS equations. Though there exists an AGS code, which was used for calculating (d,p) reactions on Carbon-12, this code needs to not only be modernized, but also extended for heavier nuclei. The most efficient way to proceed here is essentially a redesign of the code, specifically having in mind that we plan to take advantage of today's parallel architectures.

We will also focus on the two-body input (i.e. the NN and NA interactions) to the AGS equations, specifically the optical potentials for the neutron- and proton-nucleus interactions. There is essentially no literature for nuclei heavier than Carbon-12, on separable optical potentials. We plan to use the Fourier transform of a Wood-Saxon optical potential as starting point for the form factors, and develop numerical fit routines to determine the strength and range parameters for arbitrary partial waves. Preliminary work in this directions is described in Sec. 2.10.3.

\subsection{Transfers to Resonances}

Personnel involved: A.M. Mukhamdezhanov, J.E. Escher, I.J. Thompson, and G. Arbanas 
One important activity of the TORUS collaboration is the development of a theoretical description of stripping to resonances that provides valuable insights into the structure of the resonant state. The necessity for theoretical advances in this field is due to the fact that the number of the experiments leading to population of the resonance states is mounting, but there are no good descriptions and readily-available codes that can be used to analyze the data.

There are two main reasons for the lack of a practical theory of stripping to resonance states that could be used by experimental groups: the numerical problem of the convergence of the distorted-wave Born approximation (DWBA) matrix element when the full transition operator is included, and the ambiguity over what spectroscopic information can be extracted from the analysis of transfer reactions populating the resonance states. Since the resonant binary amplitudes in the very popular $R$-matrix approach are parameterized in terms of the observable partial resonance widths, it is quite natural and useful to pursue a theory in which the transfer cross sections are parameterized in terms of the same observables as in applications to binary resonance reactions.

In April 2011, A.M.M. initiated work on a new approach to deuteron stripping reactions that populate resonance states. This approach is based on the surface integral formulation of the reaction theory, outlined in [14], and a generalized $R$-matrix approach. The formalism was first developed for the DWBA and subsequently extended to the continuum discretized coupled channels (CDCC) approach, which is the ultimate goal of this work. The CDCC wave function takes into account not only the initial elastic $d+A$ channel but also its coupling to the deuteron breakup channel $p+n+A$ that is absent in the DWBA. Stripping to both bound states and resonances can be treated in this formalism.

The reaction amplitude is expressed in terms of the resonance partial widths, boundary conditions and channel radii, i.e. exactly the same quantities that appear in the analysis of binary resonance reactions in the $R$-matrix approach. This makes it, in principle, possible to simultaneously analyze deuteron stripping to resonance states and binary resonance reactions. An important step in the derivation of the reaction amplitude is the split of the configuration space of the subsystem "transferred nucleon-target" into the internal and external regions, similarly to what is being done in the $R$-matrix approach. In the post CDCC approach the reaction amplitude is given by the surface integral and the internal post form CDCC amplitude, which is expected to be small compared to the surface integral. The absence of the external amplitude in the CDCC approach solves the convergence problem. The expression for the amplitude has been derived for the general two-channel, multilevel-case and will allow one to analyze practically all possible cases, including sub-threshold resonances. The theory provides a consistent tool to analyze both binary resonant reactions and deuteron stripping in terms of the same parameters. Thus, for the first time, the theory of the deuteron stripping to resonance has been developed in a form that allows experimentalists to analyze experimental data and obtain resonance partial widths. A summary of the work was published in [17]. This work is also important for planing and interpreting inverse-kinematics experiments with radioactive beams and for extracting the relevant nuclear-structure information from the measurements. In particular, $(d, p)$ experiments on unstable nuclei are being planned at our local institutions at FRIB and at the Cyclotron Institute, Texas A\&M University. The TORUS collaboration has begun to systematically test the approximations introduced in this approach. Initial results are very encouraging (see Section 2.1) and pave the way for implementing this method in a new code. 
The newly-developed theory for deuteron stripping to resonance states has been applied to the analysis of the Trojan Horse (TH) resonance reactions. The only difference between the classical deuteron stripping and the Trojan Horse method (THM) is that in the former only the outgoing nucleon-spectator is measured while in the latter both fragments formed after the resonance decay are measured in the coincidence. Because the THM provides a powerful indirect technique in nuclear astrophysics, delivering the Trojan Horse method theory is another important extension of the deuteron stripping reactions to resonance states as indirect tool to determine the astrophysical factors for the $(n, \alpha)$ and $(p, \alpha)$ resonant reactions. The first application of the developed surface integral formalism in the plane wave approximation for the reaction ${ }^{19} \mathrm{~F}(p, \alpha){ }^{16} \mathrm{O}$ has been presented in [8].

Year 3 plan: We will incorporate the formalism developed by A.M. Mukhamedzhanov into a modified Fresco code. This will be tested by various test applications, e.g. partial widths will be extracted that can be compared with known results from $(\mathrm{n}, \alpha),(\mathrm{p}, \alpha)$ and $(\mathrm{n}, \gamma)$ reactions. The effects of deuteron breakup in the initial channel will be considered using the CDCC approach. We will also investigate the possibility to simultaneously fit resolved resonance parameters and the smoothly varying cross section, which would be a useful development for the nuclear data community. This work will be carried out by A.M. Mukhamedzhanov, J.E. Escher, I.J. Thompson, and G. Arbanas.

\subsection{Capture Reactions}

Personnel involved: G. Arbanas and I.J. Thompson

\subsubsection{Semi-direct capture mechanisms}

It was recognized in the 1970's that semidirect capture, that is a two-step process that excites a giant-dipole resonance in the first step, followed by its radiative de-excitation in the second step, is a dominant process near giant-dipole resonances in the range 5-20 MeV. At energies below $1 \mathrm{MeV}$ that are of astrophysical interest, contributions from semidirect processes are smaller than those of direct capture, but have nevertheless been found by model computations to be as high as $50 \%$ on nickel. This motivated us to implement semidirect capture in the coupled-channel code Cupido.

For medium and heavy mass nuclei away from closed shells, the statistical (i.e. compound) capture processes are expected to dominate. This hypothesis motivated measurement of singleparticle levels in ${ }^{131} \mathrm{Sn}$ that is near a closed shell $N=82$, by measuring ${ }^{130} \mathrm{Sn}(\mathrm{d}, \mathrm{p}){ }^{131} \mathrm{Sn}$ in inverse kinematics. Therefore, our first practical applications of direct-semidirect capture has been to ${ }^{130} \mathrm{Sn}(\mathrm{n}, \gamma){ }^{131} \mathrm{Sn}$.

To assess relative contribution of direct-semidirect vs. compound capture on ${ }^{130} \mathrm{Sn}(\mathrm{n}, \gamma){ }^{131} \mathrm{Sn}$, we computed statistical capture cross section using the Hauser-Feshbach code TALYS. Paritydependent level-density model of TALYS was used in this computation, to account for any effects that parity may have on statistical capture below $1 \mathrm{MeV}$ where the effects of parity-dependent level density on capture are expected to be the largest. Comparison of parity-dependent and parityindependent level densities reveals a difference of at most 30\%. Parity-dependence in level density was found to be relatively small even at low energies, and the overall level density was found to 
relatively large because the $h_{11 / 2}$ single-particle level that is below the $\mathrm{N}=80$ allows for a large number of negative parity-states even at low excitation energies. A large level density makes a statistical capture large.

Due to this large level density, the statistical neutron capture on ${ }^{130} \mathrm{Sn}$ computed by TALYS was found to be an order of magnitude larger than the direct-semidirect capture computed by FRESCO. Based on our computations and also on those of Chiba et al., DSD capture will be larger than statistical for tin isotopes with mass $A \geq 130$. The semidirect capture via GDR was found to be negligible for neutron energies below $1 \mathrm{MeV}$, and this is likely to be true for other tin isotopes in this mass range.

\subsubsection{Identifying a need for better reactions model in nuclear astrophysics(?)}

Based on prior systematic computations of capture reactions rates, i.e. Maxwellian averaged capture cross section at temperature of $30 \mathrm{keV}$, cross sections computed by Hauser-Feshbach method, a comparison with corresponding rates computed on measured experimental data reveals disagreement in some cases by factors on the order of 2-3 or more. The magnitude of these discrepancies suggests a need for an improved neutron capture model. We note that the computation of astrophysical neutron capture rates involves energy-averaging of HF capture cross section weighted by a Maxwellian distribution function at $\mathrm{T}=30 \mathrm{keV}$. The width of the Maxwellian distribution function is $100 \mathrm{keV}$ wide, while optical transmission coefficients that are used in the HF model are a priori energy-averaged over $1 \mathrm{MeV}$.

This inconsistency between the two energy averaging intervals, $100 \mathrm{keV}$ vs. $1 \mathrm{MeV}$, may introduce inaccuracy into computation of neutron capture rates. The magnitude of this inaccuracy could be estimated by (numerically) energy-averaging experimentally measured cross section with $100 \mathrm{keV}$ and $1 \mathrm{MeV}$ and then computing their respective capture rates, and comparing these to the rate obtained using the un-averaged fine resolution cross section. A variance among the capture rates obtained in these three ways could be used as an estimate (upper limit) of improvement in theoretical accuracy that could be hoped for by improving energy resolution of theoretical capture computations from $1 \mathrm{MeV}$ down to $100 \mathrm{keV}$.

Year 3 plan: Determine if the accuracy in capture rates could be improved, especially in the treatment of doorway resonances.

\subsubsection{Two-hole pair structure of the ${ }^{130} \mathrm{Sn}$ ground state}

The two holes in the ground state of ${ }^{130} \mathrm{Sn}$ are strongly paired. This pairing wave-function is consistent with there being no significant stripping to $d_{3 / 2}$ seen in the ${ }^{130} \mathrm{Sn}(\mathrm{d}, \mathrm{p}){ }^{131} \mathrm{Sn}$ experiment. (Stripping to the $h_{11 / 2}$ is expected to be small due to its high angular momentum.) We applied an exactly solvable pairing Hamiltonian found by Richardson (and successfully applied to reproduce the spectrum of ${ }^{206} \mathrm{~Pb}$ ) to ${ }^{130} \mathrm{Sn}$. In the process, a generalization of the Richardson's model to a separable pairing interaction strength was found, with possible applications in nuclear structure and other fields of many-body physics. The energy of the first non-collective excited state in ${ }^{130} \mathrm{Sn}$ is at $1.946 \mathrm{MeV}$ and is of spin $7^{-}$, that corresponds to breaking of the pair of holes into $h_{11 / 2}$ and $d_{3 / 2}$ (minus the $70 \mathrm{keV}$ energy difference between the single-particle levels). 


\subsection{Isobaric Analogue Resonances}

\section{Personnel involved: G. Arbanas and I.J. Thompson}

The position and width of isobaric analogue resonances in nucleon-nucleus scattering are accurate and detailed indicators of the positions of resonances and bound states with good singleparticle characters [5]. Since determining the positions of shells and shell gaps has often been the objective of experiments with unstable isotopes, measuring isobaric analogue resonances (IAR) should be modeled as well as possible by theorists in relation to proposed experiments. These IAR have the great virtue that neutron bound states, both occupied and unoccupied, can be determined in experiments that react protons on nuclei. Proton targets can be made with hydrogen. The best information about levels is determined by $\left(\mathrm{p}, \mathrm{p}^{\prime} \gamma\right)$ coincidence experiments [25]. The displacement energies of IAR also depend critically on neutron-proton density differences, so can be used to probe those densities in the surface.

We therefore implemented within our coupled-channels code FRESCO the main Lane coupling term [3]: the interaction that couples an incident proton to a neutron at a lower energy, such as a sub-threshold energy near an unoccupied single-particle state. We see doorway resonances when the neutron energy is near a bound state. At the same time, a target neutron must have changed to a proton, so it must have been in an occupied neutron state with quantum numbers such that a proton with those parameters is not Pauli blocked. We therefore extended the Lane coupledchannels formalism to follow the non-orthogonality of this neutron channel with that configuration of an inelastic outgoing proton, and the target being left in a particle-hole excited state. This is being tested for ${ }^{208} \mathrm{~Pb}$, for which good ( $\left.\mathrm{p}, \mathrm{p}^{\prime} \gamma\right)$ coincidence data exists [25], and we will soon make predictions for the equivalent processes for ${ }^{132} \mathrm{Sn}$, and talk to experimentalists about possible experiments.

Year 3 plan: We will complete testing of our treatment of isobar-analogue resonances, including multistep-transfer contributions, and compute cross sections for selected neutron-rich nuclides.

\subsection{Pair Transfers}

\section{Personnel involved: I.J. Thompson}

In the last year, we have calculated two-nucleon transfers for the $(\mathrm{p}, \mathrm{t})$ and $\left(\mathrm{p},{ }^{3} \mathrm{He}\right)$ reactions on a wide variety of nuclei using overlap form factors from the shell-model code OXBASH, in collaboration with B.A. Brown (MSU). A systematic front-end to FRESCO was written which enables the easy calculation of both simultaneous and sequential transfer reaction mechanisms.

Year 3 plan: In Year 3 (if time permits), we will calculate two-nucleon transfers for the (p,t) and $\left(\mathrm{p},{ }^{3} \mathrm{He}\right)$ reactions using overlaps from the No-core-Shell-Model and the Variational-Monte-Carlo method when available.

\subsection{Implementing Advanced Optical Potentials}

\subsubsection{Dispersive Optical Potentials}

Personnel involved: F.M. Nunes 
The dispersive optical model was applied to transfer reactions: a systematic study of (d, p) reactions on closed-shell nuclei using the finite-range adiabatic reaction model was performed [19] at several beam energies and results were compared to data as well as to predictions using a standard global optical potential. Overall, we found that the dispersive optical model was able to describe the angular distributions as well as or better than the global parametrization. In addition, it also constrained the overlap function. Spectroscopic factors extracted using the dispersive optical model are generally lower than those using standard parameters, exhibit a reduced dependence on beam energy, and are more in line with results obtained from (e,e'p) measurements.

This work was partially funded by TORUS, and partially by NSF Grant No. PHY- 0555893 and the DOE through Grant No. DE-FG52-08NA28552

\subsubsection{Microscopic Folding Optical Potentials}

\section{Personnel involved: Ch. Elster and S. Weppner}

The PI Elster and collaborator S.P. Weppner completed work started during the first grant year on developing a microscopic folding optical potential for the reaction ${ }^{6} \mathrm{He}(\mathrm{p}, \mathrm{p}){ }^{6} \mathrm{He}$, which takes into account the cluster structure of the ${ }^{6} \mathrm{He}$ nucleus.

In this work we extended the concept of a traditional single-particle folding optical potential in first order in the Watson multiple scattering expansion such that the cluster structure of a halo nucleus is incorporated. In practice, this means that we take into account the internal motion of the valance neutrons with respect to the core. In our work we concentrated on the ${ }^{6} \mathrm{He}$ nucleus, however, the formulation we introduced can be further extended to four or five-body clusters, e.g. to ${ }^{8} \mathrm{He}$. For our calculations we used the density matrix of the three-body cluster orbital shell model approximation (COSMA) introduced in Refs. [32, 31] for the ${ }^{6} \mathrm{He}$ nucleus. This density matrix is based on single harmonic oscillator wave functions for the s- and p-shell of ${ }^{6} \mathrm{He}$ and allows a straightforward calculation of the required correlation densities needed for the optical potential. The resulting folding optical potential contains a six-dimensional integration over internal vector momenta, which is calculated via Monte Carlo integration.

We calculated the angular distribution of the differential cross section and the analyzing power at $71 \mathrm{MeV}, 100 \mathrm{MeV}$, and $200 \mathrm{MeV} /$ nucleon and compared our results with experimental data at $71 \mathrm{MeV} /$ nucleon [28]. We find that the cluster model lowers the cross section for the small angles and brings it closer to the data. Though we do not describe the very small analyzing power at the small angles, we find that the cluster formulation together with a $\mathrm{NN} \mathrm{t-matrix} \mathrm{which} \mathrm{takes}$ into account a modification due to the nuclear medium is able to produce a negative analyzing power at larger angles as suggested by the data. Our predictions for the higher energies indicate that the lowering of the differential cross section for small momentum transfers (angles) using a cluster paradigm remains visible. The cluster ansatz for the optical potential continues to predict a negative analyzing power at larger momentum transfer. Eventually experimental information should be become available to see if these predictions capture the bulk of the physics of the reaction at higher energies, or if there are additional theoretical pieces necessary to understand this reaction, preferably as function of scattering energy.

This work was funded in part by DOE contract No. DE-FG02-93ER40756 with Ohio University and under contract No. DE-SC0004084 (TORUS Collaboration). 


\subsubsection{Investigating Separable Optical Potentials for Faddeev Models}

\section{Personnel involved: Ch. Elster and L. Hlope (graduate student, Ohio University)}

For the generalized AGS equations adapted to calculate $(\mathrm{d}, \mathrm{p})$ reactions for arbitrary nuclei, we proposed to use separabilized potentials between the constituents of the reaction. While there is a large body of work on separable representations of the nucleon-nucleon interaction (see e.g. [11, 4]), and some in the three-body description of light nuclei like ${ }^{6} \mathrm{He}$ [10] and ${ }^{6} \mathrm{Li}$ [9], we are not aware of similar work for heavy nuclei. The separable potentials for ${ }^{6} \mathrm{He}$ and ${ }^{6} \mathrm{Li}$ are of rank-1 and contain Yamaguchi-type form factors as they are common in the description of the NN interaction.

For describing $(\mathrm{d}, \mathrm{p})$ reactions within a Faddeev formulation one needs the interaction between the constituents in the system. For nuclear reactions this means the nucleon-nucleon interaction for the deuteron subsystem and the neutron or proton optical potentials between the neutron and proton in the deuteron and the target nucleus. A separable nucleon-carbon-12 optical potential was proposed in Ref. [15], consisting of a rank-1 Yamaguchi-type form factor fitted to the positive energies and a similar term describing the bound states in the nucleon-carbon-12 configuration. Using Yamaguchi-type form factors in rank-1 separable potentials has the definite advantage that one can establish an analytic relation between the low-energy parameters (scattering length and effective range) and the parameters of the form factor, and in case of a resonant behavior of a phase shift they can be analytically determined from the shape of the resonance, as e.g. in [10, 9]. However, it is by no means clear that Yamaguchi-type form factors are equally well suited for parameterizing optical potentials for heavier nuclei, which are phenomenologically described by Wood-Saxon rather than Yukawa-type potentials.

Using as test case phenomenological Wood-Saxon type optical potential for $n+{ }^{48} \mathrm{Ca}$, PI Elster and graduate student L. Hlope started investigating form factors of different shapes. We first looked at a series approximation to a Wood-Saxon potential from Ref. [24], which is suggested to converge very quickly and where the Fourier transforms of the terms have an analytic form simple enough to analytically connect the parameters in the functions to the low energy scattering parameters similar to the Yamaguchi form factors. We constructed a separable s-wave potential with this form factor, compared it with a Yamaguchi form factor fitted to the same low energy parameters. We found that both functions not only give the same on-shell values (as they should by construction), they also have the exactly the same shape for all momenta. These findings are for the real part of the optical potential. We conclude that the suggestion of Ref. [24] does not lead to anything new, though the parameters can be related to the traditional Wood-Saxon parameters. Next we will study the exact Fourier transform of the Wood-Saxon potential [12], which however does not have an analytic partial wave expressions. Here parameters can only be determined numerically. Our final goal is to have not only form factors which allow the description of partial waves with a low rank (best would be rank-1), but also have a numerical fit procedure to obtain the parameters of those form factors with relative ease.

This work will be continued through the end of the 2nd year funding period and the beginning of the 3rd year funding period, and is supported in part by TORUS and in part by DOE contract No. DE-FG02-93ER40756.

Year 3 plan: Determine low-rank separable expansions for optical potentials, including Coulomb, imaginary and spin-orbit components. 


\section{Project Management}

\section{Coordination}

- The coordinating P.I. coordinates the different sub-projects, and ensures the cohesion of the overall project.

- Monthly conference calls ensure that practical information is exchanged, and that research projects, visitors and collaborations are properly coordinated.

- Additional conference calls are set up as needed, and our website (see below) is used to deposit internal documents for discussion.

- Collaborative visits and small-group conference calls held on a regular basis to allow for detailed discussions of physics issues.

\section{Website}

We have developed a website at http://www.reactiontheory.org that is hosted at MSU. For the public, this site contains general information about our collaboration, our research papers and talks, the workshops and conferences we attend, and lists of relevant experiments.

For ourselves (protected by a password), we have information about our budget, our plans and deliverables, minutes from our meetings and conference calls, and also a place to deposit internal documents for access by the collaboration.

\section{Collaborative visits in Year 1Q4 and Year 2Q1-3}

April 2011: Ch. Elster and N. Upadhyay visited TAMU

May 2011: I.J. Thompson, F. Nunes and J. Escher attended the ECT* workshop "Recent Developments in Transfer and Knockout Reactions"

July 2011 : N. Upadhyay visited OU

June 2011: the whole collaboration met at MSU for annual meeting and DOE review

Oct 2011: I.J. Thompson, A. Mukhamedzhanov, C. Elster and F. Nunes had a collaboration meeting during the DNP meeting at MSU

Oct 2011: Ch. Elster visited MSU

Nov 2011: Ch. Elster visited MSU

Dec 2011: I.J. Thompson visited MSU 


\section{Postdoctoral Staff and Visits}

\section{TORUS Postdoctoral researcher}

Year 2 report: During the first part of the year, Neelam Upadhyay concluded the CDCC calculations regarding the breakup of deuterons for a number of test cases to compare with exact Faddeev (see Section 2.2) and collaborated in writing up the results [29]. She also started to study an existing AGS code (visit to Texas in April 2011) and learning how to use separable interactions (visit to Ohio in July 2011). More recently Neelam has been meticulously deriving the general form for the equations to be implemented by our collaboration and has been comparing several techniques to handle the Coulomb interactions in momentum space. Her present task is to implement a simple study case to explore the method introduced by Oryu [23].

Neelam had the opportunity to present her work at the DNP meeting (Fall 2011) and at a seminar at NSCL in Feb 2012. She participated in a Winter School on computational nuclear physics in Jan 2012, at ORNL and has been sitting through Nuclear Reaction lectures (phy982) by Prof. Nunes at MSU.

Finally, she also initiated a collaboration with an experimentalist Anissa Bey from Univ. Tennessee regarding the analysis of data obtained at ORNL. This work will be published in the near future.

\section{External Visitors in Year 1Q4 and Year 2Q1-3}

- Prof. S.P. Weppner spent his sabbatical in the academic year 2010-11 from Eckerd College (FL) at Ohio University, for the work of his stay was provided by Ohio University (Institute of Nuclear and Particle Physics, and Department of Physics and Astronomy) and a small portion by the U.S. Department of Energy under Grant No. DE-FG02-93ER40756.

\section{Other Collaborators in Year 1Q4 and Year 2Q1-3}

These collaborations contributed to our project, but were not funded by this grant:

- F.S. Dietrich (LLNL)

- B.A. Brown (MSU)

- R. Kozub (Tenn. Tech. Univ.)

- A.K. Kerman (MIT)

\section{Planned Visitors in Year 2Q4 and Year 3}

We plan to support the visits of the following people in Year 3 as visitors or consultants:

- Peter C. Tandy (Kent State U., OH) visit to OU

- Steve Weppner (Eckerd College, FL) visit to OU

- Rimas Lazauskas to visit MSU

- Arnas Deltuva to visit MSU 


\section{Plans for Year 3}

1. We will determine the size of exterior prior and interior post contributions for transfers to a wide range of resonances, to predict the accuracy of the Surface Approximation method of Section 2.4. We want to confirm in particular the smallness of the exterior prior contributions within the Continuum Discretized Coupled-Channels (CDCC) approach.

2. Investigate whether the rearrangement triangle diagram containing the off-shell proton-target Coulomb scattering amplitude can be replaced by the renormalized pole diagram describing the proton transfer.

3. Treatment and practical implementation of the Coulomb potential in the AGS equations.

4. We will start the actual coding of the AGS equations.

5. Determine if the discretization accuracy in capture rates can be improved, especially in the treatment of doorway resonances

6. We will complete testing of our treatment of isobar-analogue resonances, including multisteptransfer contributions, and compute cross sections for selected neutron-rich nuclides.

7. In Year 3 (if time permits), we will calculate two-nucleon transfers for the $(\mathrm{p}, \mathrm{t})$ and $\left(\mathrm{p},{ }^{3} \mathrm{He}\right)$ reactions using overlaps from the No-core-Shell-Model and the Variational-Monte-Carlo method when available.

8. Determine low-rank separable expansions for optical potentials, including Coulomb, imaginary and spin-orbit components. 


\section{Deliverables}

\subsection{Publications}

1. Published paper [22]: Phys. Rev. C 83, 034610 (2011) - Published March 22, 2011 Improved description of ${ }^{34,36,46} \mathbf{A r}(\mathbf{p}, \mathbf{d})$ transfer reactions

F. M. Nunes, A. Deltuva, and June Hong.

An improved description of single neutron stripping from ${ }^{34,36,46} \mathrm{Ar}$ beams at $33 \mathrm{MeV} /$ nucleon by a hydrogen target is presented and the dependence on the neutron-proton asymmetry of the spectroscopic factors is further investigated. A finite range adiabatic model is used in the analysis and compared to previous zero range and local energy approximations. Full three-body Faddeev calculations are performed to estimate the error in the reaction theory. In addition, errors from the optical potentials are also evaluated. From our new spectroscopic factors extracted from transfer, it is possible to corroborate the neutron-proton asymmetry dependence reported from knockout measurements.

2. Published paper [13]: Phys. Rev. C 84, 034601 (2011) - Published September 1, 2011 Direct reaction measurements with a ${ }^{132} \mathrm{Sn}$ radioactive ion beam

K. L. Jones, F. M. Nunes, et al.

The $(\mathrm{d}, \mathrm{p})$ neutron transfer and $(\mathrm{d}, \mathrm{d})$ elastic scattering reactions were measured in inverse kinematics using a radioactive ion beam of ${ }^{132} \mathrm{Sn}$ at $630 \mathrm{MeV}$. The elastic scattering data were taken in a region where Rutherford scattering dominated the reaction, and nuclear effects account for less than $8 \%$ of the elastic scattering cross section. The magnitude of the nuclear effects, in the angular range studied, was found to be independent of the optical potential used, allowing the transfer data to be normalized in a reliable manner. The neutron-transfer reaction populated a previously unmeasured state at $1363 \mathrm{keV}$, which is most likely the single-particle $3 p_{1 / 2}$ state expected above the $\mathrm{N}=82$ shell closure. The data were analyzed using finite-range adiabatic-wave calculations and the results compared with the previous analysis using the distorted-wave Born approximation. Angular distributions for the ground and first-excited states are consistent with the previous tentative spin and parity assignments. Spectroscopic factors extracted from the differential cross sections are similar to those found for the one-neutron states beyond the benchmark doubly magic nucleus ${ }^{208} \mathrm{~Pb}$.

3. Publishes paper [27]: Phys. Rev. C 84, 035805 (2011) - Published September 14, 2011

\section{Asymptotic normalization of mirror states and the effect of couplings}

L. J. Titus, P. Capel, and F. M. Nunes.

Assuming that the ratio between asymptotic normalization coefficients of mirror states is model independent, charge symmetry can be used to indirectly extract astrophysically relevant proton capture reactions on proton-rich nuclei based on information on stable isotopes. The assumption has been tested for light nuclei within the microscopic cluster model. In this work we explore the Hamiltonian independence of the ratio between asymptotic normalization coefficients of mirror states when deformation and core excitation is introduced in the system. For this purpose we consider a phenomenological rotor $+\mathrm{N}$ model where the valence nucleon is subject to a deformed mean field and the core is allowed to excite. We apply the model to ${ }^{8} \mathrm{Li} /{ }^{8} \mathrm{~B},{ }^{13} \mathrm{C} /{ }^{13} \mathrm{~N},{ }^{17} \mathrm{O} /{ }^{17} \mathrm{~F},{ }^{23} \mathrm{Ne} /{ }^{23} \mathrm{Al}$, and ${ }^{27} \mathrm{Mg} /{ }^{27} \mathrm{P}$. Our results show that, for most studied cases, the ratio between asymptotic normalization coefficients of mirror states is independent of the strength and multipolarity of the couplings induced. The exception is for cases in which there is an s-wave coupled to the ground state of the core, the proton 
system is loosely bound, and the states have large admixture with other configurations. We discuss the implications of our results for novae.

4. Published paper [21]: Phys. Rev. C 84, 034607 (2011) - Published September 19, 2011 Adiabatic approximation versus exact Faddeev method for $(d, p)$ and $(p, d)$ reactions F. M. Nunes and A. Deltuva.

The finite-range adiabatic wave approximation (ADWA) provides a practical method to analyze (d, p) or $(\mathrm{p}, \mathrm{d})$ reactions; however, until now the level of accuracy obtained in the description of the reaction dynamics has not been determined. In this work, we perform a systematic comparison between the finite-range adiabatic wave approximation and the exact Faddeev method. We include studies of ${ }^{11} \mathrm{Be}(\mathrm{p}, \mathrm{d}){ }^{10} \mathrm{Be}$ (g.s.) at $\mathrm{Ep}=5,10$, and $35 \mathrm{MeV} ;{ }^{12} \mathrm{C}(\mathrm{d}, \mathrm{p}){ }^{13} \mathrm{C}$ (g.s.) at $\mathrm{Ed}=7,12$, and $56 \mathrm{MeV}$; and ${ }^{48} \mathrm{Ca}(\mathrm{d}, \mathrm{p}){ }^{49} \mathrm{Ca}$ (g.s.) at $\mathrm{Ed}=19,56$, and $100 \mathrm{MeV}$. Results show that the two methods agree within $\sim 5$ $\%$ for a range of beam energies $\left(E_{d}=20-40 \mathrm{MeV}\right)$ but differences increase significantly for very low energies and for the highest energies. Our tests show that ADWA agrees best with the Faddeev method when the angular momentum transfer is small $? 1=0$ and when the neutron-nucleus system is loosely bound.

5. Published paper [19]: Phys. Rev. C 84, 044611 (2011) - Published October 14, 2011

\section{Transfer reactions and the dispersive optical model}

N. B. Nguyen, S. J. Waldecker, F. M. Nunes, R. J. Charity, and W. H. Dickhoff.

The dispersive optical model is applied to transfer reactions. A systematic study of (d, p) reactions on closed-shell nuclei using the finite-range adiabatic reaction model is performed at several beam energies and results are compared to data as well as to predictions using a standard global optical potential. Overall, we find that the dispersive optical model is able to describe the angular distributions as well as or better than the global parametrization. In addition, it also constrains the overlap function. Spectroscopic factors extracted using the dispersive optical model are generally lower than those using standard parameters, exhibit a reduced dependence on beam energy, and are more in line with results obtained from (e,e'p) measurements.

6. Published paper [20]: Proceedings CGS14, World Scientific, in press. Are present reaction theories for studying rare isotopes good enough? F. M. Nunes, P. Capel, R.J. Charity, A. Deltuva, W.Dickhoff, H. Esbensen, R.C. Johnson, N.B. Nguyen, N.J. Upadhyay, S.J. Waldecker..

Rare isotopes are most often studied through nuclear reactions. Nuclear reactions can be used to obtain detailed structure information but also in connection to astrophysics to determine specific capture rates. In order to extract the desired information it is crucial to have a reliable framework that describes the reaction process accurately. A few recent developments for transfer and breakup reactions will be presented. These include recent studies on the reliability of existing theories as well as effort to reduce the ambiguities in the predicted observables.

7. Published paper [7]: Physics Letters B 705, 112 (2011) - Published September 28, 2011

\section{One-neutron halo structure by the ratio method}

P. Capel, R. C. Johnson, F. M. Nunes

We present a new observable to study halo nuclei. This new observable is a particular ratio of angular distributions for elastic breakup and scattering. For one-neutron halo nuclei, it is shown to be independent of the reaction mechanism and to provide significant information about the structure of the 
projectile, including binding energy, partial-wave configuration, and radial wave function of the halo. This observable offers new capabilities for the study of nuclear structure far from stability.

8. Published paper [18]: Phys. Rev. C 83, 055805 (2011) - Published May 31, 2011. Reexamination of the astrophysical $\mathbf{S}$ factor for the $\alpha+d \rightarrow^{6} \mathrm{Li}+\gamma$ reaction, A. M. Mukhamedzhanov, L. D. Blokhintsev and B. F. Irgaziev. Recently to study the radiative capture $\alpha+d \rightarrow{ }^{6} \mathrm{Li}+\gamma$ process a new measurement of the ${ }^{6} \mathrm{Li}(\mathrm{A} 150 \mathrm{MeV})$ dissociation in the field of ${ }^{208} \mathrm{~Pb}$ has been reported in [F. Hammache et al. Phys. Rev C82, 065803 (2010)]. However, the dominance of the nuclear breakup over the Coulomb one prevented from obtaining the information about the $\alpha+d \rightarrow{ }^{6} \mathrm{Li}+\gamma$ process from the breakup data. The astrophysical $S_{24}(E)$ factor has been calculated within the $\alpha-d$ two-body potential model with potentials determined from the fits to the $\alpha-d$ elastic scattering phase shifts. However, the scattering phase shift itself doesn't provide a unique $\alpha-d$ bound state potential, which is the most crucial input when calculating the $S_{24}(E)$ astrophysical factor at astrophysical energies. In this work we emphasize an important role of the asymptotic normalization coefficient (ANC) for ${ }^{6} \mathrm{Li} \rightarrow \alpha+d$, which controls the overall normalization of the peripheral $\alpha+d \rightarrow{ }^{6} \mathrm{Li}+\gamma$ process and is determined by the adopted $\alpha-d$ bound state potential. We demonstrate that the ANC previously determined from the $\alpha-d$ elastic scattering $s$-wave phase shift in [Blokhintsev et. al Phys. Rev. C 48, 2390 (1993)] gives $S_{24}(E)$, which is at low energies about 38\% lower than the one reported in [F. Hammache et al. Phys. Rev C82, 065803 (2010)]. We recalculate also the reaction rates, which are also lower than those obtained in [F. Hammache et al. Phys. Rev C82, 065803 (2010)].

9. Published paper [16]: Physical Review C 84, 024616 (2011) - Published August 29 , 2011. Asymptotic normalization coefficients from the ${ }^{14} \mathbf{C}(\mathbf{d}, \mathbf{p})^{15} \mathbf{C}$ reaction,

A.M. Mukhamedzhanov, V. Burjan, M. Gulino, Z. Hons, V. Kroha, M. McCleskey, J. Mrazek, N. Nguyen, F. M. Nunes, S. Piskor, S. Romano, M.A. L. Sergi, C. Spitaleri, and R. E. Tribble.

The ${ }^{14} \mathrm{C}(\mathrm{n}, \gamma){ }^{15} \mathrm{C}$ reaction plays an important role in inhomogeneous big bang models. In Timofeyuk et al. [Phys. Rev. Lett. 96162501 (2006)] it was shown that the ${ }^{14} \mathrm{C}(\mathrm{n}, \gamma){ }^{15} \mathrm{C}$ radiative capture at astrophysically relevant energies is a peripheral reaction, i.e., the overall normalization of its cross section is determined by the asymptotic normalization coefficient (ANC) for ${ }^{14} \mathrm{C} \rightarrow{ }^{14} \mathrm{C}+\mathrm{n}$. Here we present new measurements of the ${ }^{14} \mathrm{C}(\mathrm{d}, \mathrm{p}){ }^{15} \mathrm{C}$ differential cross sections at deuteron incident energy of $17.06 \mathrm{MeV}$ and the analysis to determine the ANCs for neutron removal from the ground and first excited states of ${ }^{15} \mathrm{C}$. The results are compared with previous estimations.

10. Published paper [17]: Phys. Rev. C 84, 044616 (2011) - Published October 21, 2011.

Theory of deuteron stripping: From surface integrals to a generalized R-matrix approach,

A. M. Mukhamedzhanov.

There are two main reasons for absence of the practical theory of stripping to resonance states which could be used by experimental groups: numerical problem of the convergence of the DWBA matrix element when the full transition operator is included and it is unclear what spectroscopic information can be extracted from the analysis of transfer reactions populating the resonance states. The purpose of this paper is to address both questions. The theory of the deuteron stripping is developed, which is based on the post continuum discretized coupled channels (CDCC) formalism going beyond of the DWBA and surface integral formulation of the reaction theory [A. S. Kadyrov et al., Ann. Phys. 324, 
1516 (2009)]. First, the formalism is developed for the DWBA and then extended to the CDCC formalism, which is ultimate goal of this work. The CDCC wave function takes into account not only the initial elastic $d+A$ channel but also its coupling to the deuteron breakup channel $p+n+A$ missing in the DWBA. Stripping to both bound states and resonances are included. The convergence problem for stripping to resonance states is solved in the post CDCC formalism. The reaction amplitude is parametrized in terms of the reduced width amplitudes (ANCs), inverse level matrix, boundary condition and channel radius, that is the same parameters which are used in the conventional $R$-matrix method. For stripping to resonance states many-level, one and two-channel cases are considered. The theory provides a consistent tool to analyze both binary resonant reactions and deuteron stripping in terms of the same parameters.

11. Published paper [8]: The Astrophysical Journal Letters, 739, L54 (2011) - Published September 9, 2011.

The fluorine destruction in stars: first experimental study of the ${ }^{19} \mathrm{~F}\left(p, \alpha_{0}\right){ }^{16} \mathrm{O}$ reaction at astrophysical energies,

M. La Cognata, A. M. Mukhamedzhanov, C. Spitaleri, I. Indelicato, M. Aliotta, V. Burjan, S. Cherubini, A. Coc, M. Gulino, Z. Hons, G. G. Kiss, V. Kroha, L. Lamia, J. Mrazek, S. Palmerini, S. Piskor, R. G. Pizzone, S. M. R. Puglia, G. G. Rapisarda, S. Romano, M. L. Sergi, and A. Tumino.

The ${ }^{19} \mathrm{~F}(p, \alpha){ }^{16} \mathrm{O}$ reaction is an important fluorine destruction channel in the proton-rich outer layers of asymptotic giant branch (AGB) stars and it might also play a role in hydrogen-deficient post-AGB star nucleosynthesis. So far, available direct measurements do not reach the energy region of astrophysical interest $\left(E_{c m} \lesssim 300 \mathrm{keV}\right.$ ), because of the hindrance effect of the Coulomb barrier. The Trojan Horse (TH) method was thus used to access this energy region, by extracting the quasi-free contribution to the ${ }^{2} \mathrm{H}\left({ }^{19} \mathrm{~F}, \alpha^{16} \mathrm{O}\right) \mathrm{n}$ and the ${ }^{19} \mathrm{~F}\left({ }^{3} \mathrm{He}, \alpha^{16}\right) \mathrm{d}$ reactions. The TH measurement of the $\alpha_{0}$ channel shows the presence of resonant structures not observed before, which cause an increase of the reaction rate at astrophysical temperatures up to a factor of 1.7 , with potential consequences for stellar nucleosynthesis.

\subsection{Preprints and Reports}

1. Submitted to Phys. Rev. Lett. [26]:

Halo nucleus ${ }^{11}$ Be: a spectroscopic study via neutron transfer

K.T. Schmitt, K.L Jones, A. Bey, S.H. Ahn, D.W. Bardayan, J.C. Blackmon, S. Brown, K.Y. Chae, K.A. Chipps, J.A. Cizewski, K. I. Hahn, J.J. Kolata, R.L. Kozub, J.F. Liang, C. Matei, M. Matos, D. Matyas, B. Moazen, C. Nesaraja, F.M. Nunes, P.D. O’Malley, S.D. Pain, W.A. Peters, S.T. Pittman, A. Roberts, D. Shapira, J.F. Shriner Jr., M.S. Smith, I. Spassova, D.W. Stracener, A.N. Villano and G. Wilson.

Despite ${ }^{11} \mathrm{Be}$ being the best known example of a one-neutron halo nucleus, and one of the most heavily-studied nuclei in recent years, the spectroscopic factors for the two bound states are exceedingly poorly constrained. The $(\mathrm{d}, \mathrm{p})$ reaction has been performed in inverse kinematics using a beam of the long-lived isotope ${ }^{10} \mathrm{Be}$ to study both the ground state and the unusual bound excited state halo in ${ }^{11} \mathrm{Be}$. By performing the reaction at a number of beam energies, studying the elastic channel as well as the transfer channels, and using a theoretical framework that explicitly includes deuteron 
breakup, consistent spectroscopic factors for these two states have been extracted.

2. Submitted to Phys. Rev. C [29]: arXiv:1112.5338 [nucl-th]

\section{Testing the continuum discretized coupled channel method for deuteron induced reac- tions}

N. J. Upadhyay, A. Deltuva, F. M. Nunes.

The Continuum Discretized Coupled Channels (CDCC) method is a well established theory for direct nuclear reactions which includes breakup to all orders. Alternatively, the 3-body problem can be solved exactly within the Faddeev formalism which explicitly includes breakup and transfer channels to all orders. With the aim to understand how CDCC compares with the exact 3-body Faddeev formulation, we study deuteron induced reactions on: i) ${ }^{10} \mathrm{Be}$ at $E_{\mathrm{d}}=21.4,40.9$ and $71 \mathrm{MeV}$; ii) ${ }^{12} \mathrm{C}$ at $E_{\mathrm{d}}=12$ and $56 \mathrm{MeV}$; and iii) ${ }^{48} \mathrm{Ca}$ at $E_{\mathrm{d}}=56 \mathrm{MeV}$. We calculate elastic, transfer and breakup cross sections. Overall, the discrepancies found for elastic scattering are small with the exception of very backward angles. For transfer cross sections at low energy $\sim 10 \mathrm{MeV} / \mathrm{u}, \mathrm{CDCC}$ is in good agreement with the Faddeev-type results and the discrepancy increases with beam energy. On the contrary, breakup observables obtained with CDCC are in good agreement with Faddeev-type results for all but the lower energies considered here.

3. Submitted to Phys. Rev. C [6]:

\section{Comparing non-perturbative models of the breakup of neutron-halo nuclei}

P. Capel, H. Esbensen and F.M. Nunes.

Breakup reactions of loosely-bound nuclei are often used to extract structure and/or astrophysical information. Here we compare three non-perturbative reaction theories often used when analyzing breakup experiments, namely the continuum discretized coupled channel model, the time-dependent approach relying on a semiclassical approximation, and the dynamical eikonal approximation. Our test case consists of the breakup of ${ }^{15} \mathrm{C}$ on $\mathrm{Pb}$ at $68 \mathrm{MeV} /$ nucleon and $20 \mathrm{MeV} /$ nucleon.

4. Submitted to Phys. Rev. C. [30]: arXiv:1107.1816 [nucl-th]

Elastic Scattering of ${ }^{6}$ He based on a Cluster Description, S. P. Weppner and C. Elster. Elastic scattering observables (differential cross section and analyzing power) are calculated for the reaction ${ }^{6} \mathrm{He}(\mathrm{p}, \mathrm{p})^{6} \mathrm{He}$ at projectile energies starting at $71 \mathrm{MeV} /$ nucleon. The optical potential needed to describe the reaction is derived describing ${ }^{6} \mathrm{He}$ in terms of a ${ }^{4} \mathrm{He}$-core and two neutrons. The Watson first order multiple scattering ansatz is extended to accommodate the internal dynamics of a composite cluster model for the ${ }^{6} \mathrm{He}$ nucleus scattering from a nucleon projectile. The calculations are compared with the recent experiments at the projectile energy of $71 \mathrm{MeV} /$ nucleon. In addition, differential cross sections and analyzing powers are calculated at selected higher energies.

\subsection{Presentations}

1. Reactions with deuterons within the CDCC formalism, Contributed Talk by Neelam Upadhyay, APS meeting, April 2011.

2. Nuclear reactions for astrophysics and other applications, Invited Talk by Jutta Escher, Notre Dame University, March 2011.

3. Nuclear reactions for astrophysics and other applications, Invited Talk by Jutta Escher, Argonne National Laboratory, March 2011. 
4. Uncertainties from theory on transfer reactions, Invited Talk by Filomena Nunes, ECT* Workshop on Transfer and Knockout Reactions, Trento, Italy, May 9, 2011

5. Compound nucleus production by partial fusion in $(d, p)$ reactions, Invited Talk by Ian Thompson, ECT* Workshop on Transfer and Knockout Reactions, Trento, Italy, May 13, 2011

6. Using transfer and inelastic scattering mechanisms to infer compound reaction cross sections, Invited Talk by Jutta Escher, ECT* Workshop on Transfer and Knockout Reactions, Trento, Italy, May 2011.

7. Short-range correlations, nuclear reactions and spectroscopic information, Invited talk by Akram Mukhamedzhanov, ECT* Workshop on Nuclear Many-Body Open Quantum Systems: Continuum and Correlations in Light Nuclei, Trento, Italy, June 6-10, 2011.

8. Advancing the theory of transfer reactions, Invited Talk by Filomena Nunes, UNEDF annual meeting, East Lansing, 21st June 2011

9. The TORUS project, Invited Talk by Filomena Nunes, FRIB theory workshop, INT, Seattle, 8th August 2011

10. Theory and Calculation of Two-nucleon Transfer Reactions, Invited Talk by Ian Thompson, FRIB theory workshop, INT, Seattle, 8th August 2011

11. Application of Three-Body Methods in Nuclear Reactions: ${ }^{6} \mathrm{He}(p, p){ }^{6} \mathrm{He}$, Inv. Talk, Ch. Elster, INT workshop on 'Interfaces between Nuclear Reactions and Structure', August 8 September 11, 2011, Seattle, WA.

12. Are present reaction theories for studying rare isotopes good enough?, Invited Talk by Filomena Nunes, CGS14, Guelph, 2nd September 2011

13. Testing formalisms for deuteron breakup and transfer reactions, Contributed Talk by Neelam Upadhyay, DNP meeting, East Lansing, 28th October 2011.

14. Elastic Scattering of ${ }^{6}$ He based on a Cluster Description, Contributed Talk, S.P. Weppner, Ch. Elster, 2011 Fall Meeting of the APS Division of Nuclear Physics, East Lansing, MI, Bulletin of the American Physical Society,

Vol. 56, No 12, BAPS.2011.DNP.JE.2

15. Microscopic Optical Potentials for the Reaction Helium-6 (p,p) Helium-6, Invited Talk, Ch. Elster, Mini-workshop on 'Polarization Phenomena in Proton Elastic Scattering from Unstable Nuclei', December 21, 2011, RIKEN, Tokyo, Japan.

16. Microscopic Optical Potentials for the Reaction Helium-6 (p,p) Helium-6, Ch. Elster, Seminar, December 2011, RIKEN Nishina Center, Japan.

\subsection{In Preparation}

1. Theory of the Trojan Horse method, A. M. Mukhamedzhanov, to be submitted to Phys. Rev. C.

2. Reaction mechanisms of pair transfer, I. J. Thompson and B. A. Brown, for the monograph '50 Years of Nuclear BCS', Eds. R.A. Broglia and V. Zelevinsky, World Scientific. 
3. Analysis of the Trojan Horse reactions, A. M. Mukhamedzhanov, M. La Cognata, R. G. Pizzone, C. Spitaleri, to be submitted to Phys. Rev. C.

4. New insight into deuteron stripping reactions, A. M. Mukhamedzhanov, I. J. Thompson and J. Escher, to be submitted to Phys. Rev. Lett.

5. Coulomb renormalization of the ANC, A. M. Mukhamedzhanov, to be submitted to Phys. Rev. C.

6. Exactly solvable pairing Hamiltonian with state-dependent separable pairings, G. Arbanas, M. E. Pigni and A.K. Kerman, to be submitted to Phys. Rev. C.

7. Neutron single particle structure in ${ }^{131}$ Sn and direct neutron capture cross sections, R. L. Kozub, G. Arbanas, A. S. Adekola, D. W. Bardayan, J. C. Blackmon, K. Y. Chae, K. A. Chipps, J. A. Cizewski, L. Erikson, R. Hatarik, W. R. Hix, K. L. Jones, W. Krolas, F. Liang, Z. Ma, C. Matei, B. H. Moazen, C. D. Nesaraja, S. D. Pain, D. Shapira, J. F. Shriner, M. S. Smith, and T. P. Swan, to be submitted to Phys. Rev. Letts. 


\subsection{Workshops}

\subsubsection{Proposed TORUS/INT workshop}

\section{Personnel involved: J.E. Escher}

Year 2 activity and Year 3 plan: A detailed plan was developed for holding a one-week workshop at the Institute for Nuclear Theory at the University of Washington in Seattle. The focus of the proposed workshop was on deuteron-induced one-nucleon transfer reactions as a means to study the structure of and reaction with exotic nuclei. The proposal was submitted in the summer of 2011 for consideration by the INT advisory committee. It was ranked third among all proposals and tentatively scheduled for December 2012. Very recently, we were informed that the workshop can only be logistically, but not financially, supported by the INT, due to budget cuts to the Institute. Due to reduced TORUS funding and some re-allocation of funds between the TORUS institutions, we have decided to not pursue this workshop in Year 3, but postpone resubmission by one year. 


\section{References}

[1] E. O. Alt, L. D. Blokhintsev, A. M. Mukhamedzhanov, and A. I. Sattarov, Deuteron elastic scattering and stripping processes off C-12 as a three-body problem, Phys. Rev., C75 (2007), p. 054003.

[2] E. O. Alt and W. Sandhas, Scattering amplitudes and integral equations for the collision of two charged composite particles, Phys. Rev., C21 (1980), pp. 1733-1745.

[3] A.M. and Lane, Isobaric spin dependence of the optical potential and quasi-elastic $(p, n)$ reactions, Nuclear Physics, 35 (1962), pp. 676 - 685.

[4] G. H. Berthold, A. Stadler, and H. Zankel, Faddeev calculations for low-energy p-d scattering, Phys. Rev., C41 (1990), pp. 1365-1383.

[5] R. I. Betan, A. T. Kruppa, and T. Vertse, Complex energy approaches for calculating isobaric analogue states, Phys. Rev. C, 78 (2008), p. 044308.

[6] P. Capel, H. Esbensen, and F. Nunes, Comparing non-perturbative models of the breakup of neutronhalo nuclei, submitted to Phys. Rev. C., (2012).

[7] P. Capel, R. Johnson, and F. Nunes, One-neutron halo structure by the ratio method, Physics Letters B, 705 (2011), pp. $112-115$.

[8] M. L. Cognata, A. M. Mukhamedzhanov, C. Spitaleri, I. Indelicato, M. Aliotta, V. Burjan, S. Cherubini, A. Coc, M. Gulino, Z. Hons, G. G. Kiss, V. Kroha, L. Lamia, J. Mrázek, S. Palmerini, Š. Piskoř, R. G. Pizzone, S. M. R. Puglia, G. G. Rapisarda, S. Romano, M. L. Sergi, and A. Tumino, The fluorine destruction in stars: First experimental study of the ${ }^{19} F(p, \alpha){ }^{16} O$ reaction at astrophysical energies, The Astrophysical Journal Letters, 739 (2011), p. L54.

[9] A. Eskandarian and I. R. Afnan, alpha-d resonances and the low-lying states of Li-6, Phys. Rev., C46 (1992), pp. 2344-2353.

[10] A. Ghovanlou and D. R. Lehman, Separable-potential three-body model of the A=6 system. 1. He-6 ground state, Phys. Rev., C9 (1974), pp. 1730-1741.

[11] J. Haidenbauer and W. Plessas, Separable representation of the Paris nucleon nucleon potential, Phys. Rev., C30 (1984), pp. 1822-1839.

[12] R. Johnson, Validity of Born Approximation for Nuclear Scattering in Path Integral Representation, (private communication).

[13] K. L. Jones, F. M. Nunes, A. S. Adekola, D. W. Bardayan, J. C. Blackmon, K. Y. Chae, K. A. Chipps, J. A. Cizewski, L. Erikson, C. Harlin, R. Hatarik, R. Kapler, R. L. Kozub, J. F. Liang, R. Livesay, Z. Ma, B. Moazen, C. D. Nesaraja, S. D. Pain, N. P. Patterson, D. Shapira, J. F. Shriner, M. S. Smith, T. P. Swan, and J. S. Thomas, Direct reaction measurements with a ${ }^{132}$ Sn radioactive ion beam, Phys. Rev. C, 84 (2011), p. 034601.

[14] A. Kadyrov, I. Bray, A. Mukhamedzhanov, and A. Stelbovics, Surface-integral formulation of scattering theory, Annals of Physics, 324 (2009), pp. 1516 - 1546.

[15] K. Miyagawa and Y. Koike, , Prog. Theor. Phys., 82 (1989), p. 329. 
[16] A. Mukhamedzhanov, V. Burjan, M. Gulino, Z. Hons, V. Kroha, M. McCleskey, J. Mrázek, N. Nguyen, F. M. Nunes, S. Piskors, S. Romano, M. L. Sergi, C. Spitaleri, and R. E. Tribble, Asymptotic normalization coefficients from the ${ }^{14} C(d, p){ }^{15}$ C reaction, Phys. Rev. C, 84 (2011), p. 024616.

[17] A. M. Mukhamedzhanov, Theory of deuteron stripping: From surface integrals to a generalized $R$ matrix approach, Phys. Rev. C, 84 (2011), p. 044616.

[18] A. M. Mukhamedzhanov, L. D. Blokhintsev, and B. F. Irgaziev, Reexamination of the astrophysical $S$ factor for the $\alpha+d \rightarrow{ }^{6} L i+\gamma$ reaction, Phys. Rev. C, 83 (2011), p. 055805.

[19] N. B. Nguyen, S. J. Waldecker, F. M. Nunes, R. J. Charity, and W. H. Dickhoff, Transfer reactions and the dispersive optical model, Phys. Rev. C, 84 (2011), p. 044611.

[20] F. M. Nunes, P. Capel, R. Charity, A. Deltuva, W.Dickhoff, H. Esbensen, R. Johnson, N. Nguyen, N. Upadhyay, and S. Waldecker, Are present reaction theories for studying rare isotopes good enough?, Proceedings CGS14, World Scientific, in press., (2012).

[21] F. M. Nunes and A. Deltuva, Adiabatic approximation versus exact Faddeev method for (d, $p$ ) and $(p, d)$ reactions, Phys. Rev. C, 84 (2011), p. 034607.

[22] F. M. Nunes, A. Deltuva, and J. Hong, Improved description of ${ }^{34,36,46} \operatorname{Ar}(p, d)$ transfer reactions, Phys. Rev. C, 83 (2011), p. 034610.

[23] S. Oryu, Two- and three-charged-particle nuclear scattering in momentum space: A two-potential theory and a boundary condition model, Phys. Rev., C73 (2006), p. 054001.

[24] M. R. Pahlavani and R. Morad, Validity of Born Approximation for Nuclear Scattering in Path Integral Representation, (2009).

[25] E. Radermacher, M. Wilhelm, S. Albers, J. Eberth, N. Nicolay, H. Thomas, H. Tiesler, P. von Brentano, R. Schwengner, S. Skoda, G. Winter, and K. Maier, The $\gamma$-decay of particle-hole states in ${ }^{208}$ pb using the euroball cluster detector, Nuclear Physics A, 597 (1996), pp. 408 - 426.

[26] K. Schmitt, K. Jones, A. Bey, S. Ahn, D. Bardayan, J. Blackmon, S. Brown, K. Chae, K. Chipps, J. Cizewski, K. I. Hahn, J. Kolata, R. Kozub, J. Liang, C. Matei, M. Matos, D. Matyas, B. Moazen, C. Nesaraja, F. Nunes, P. O’Malley, S. Pain, W. Peters, S. Pittman, A. Roberts, D. Shapira, J. Shriner Jr., M. Smith, I. Spassova, D. Stracener, A. Villano, and G. Wilson., Halo nucleus ${ }^{11}$ be: a spectroscopic study via neutron transfer, submitted to Phys. Rev. Letts., (2012).

[27] L. J. Titus, P. Capel, and F. M. Nunes, Asymptotic normalization of mirror states and the effect of couplings, Phys. Rev. C, 84 (2011), p. 035805.

[28] T. Uesaka, S. Sakaguchi, Y. Iseri, K. Amos, N. Aoi, et al., Analyzing power for the proton elastic scattering from neutron-rich 6He nucleus, Phys.Rev., C82 (2010), p. 021602.

[29] N. Upadhyay, A. Deltuva, and F. Nunes, Testing the continuum discretized coupled channel method for deuteron induced reactions, (2011).

[30] S. Weppner and C. Elster, Elastic Scattering of ${ }^{6}$ He based on a Cluster Description, (2011).

[31] M. V. Zhukov and D. V. Fedorov, COSMA-He6, Sov. J. Nucl. Phys., 53 (1991), p. 351.

[32] M. V. Zhukov, A. A. Korsheninnikov, and M. H. Smedberg, Simplified alpha+4n model for the He-8 nucleus-COSMA, Phys. Rev., C50 (1994), pp. R1-R4. 